\title{
OCORRÊNCIA DE HEMORRAGIA PULMONAR INDUZIDA PELO EXERCÍCIO EM CAVALOS DE PÓLO NA CIDADE DO RIO DE JANEIRO/RJ
}

\author{
Juliana Nabuco Pereira Otaka', Katia Moreira Silva'1, Maria Luisa Abreu Loredo \\ Jorge $^{2}$, Carlos Alexandre Paula Gonçalves ${ }^{1}$, Denis Yukio Otaka'1, Daniel Augusto \\ Barroso Lessa ${ }^{1}$ \\ 1 UFF \\ 2 Polícia Militar RJ \\ Correspondência: Juliana Nabuco Pereira Otaka: ju.nabuco@gmail.com
}

\begin{abstract}
RESUMO: O pólo é um dos mais antigos esportes equestres e foi introduzido no Brasil na década de 1920. Doenças respiratórias são consideradas a segunda maior causa de redução de desempenho atlético, ocasionando perdas econômicas significativas em cavalos de corrida. A Hemorragia Pulmonar Induzida pelo Exercício (HPIE) é uma destas enfermidades e acomete de 40 a $90 \%$ dos animais de corrida, porém há poucos trabalhos direcionados aos cavalos de pólo. A questão é de grande preocupação para a indústria equina pelas suas implicações financeiras resultantes da queda de desempenho, perda de dias de treinamento, necessidade de medicação pré-corrida e até suspensão do animal das competições. O exame endoscópico com a utilização de um escore é o método de diagnóstico mais utilizado para quantificar a intensidade da hemorragia. Esse trabalho teve como objetivo realizar um estudo clínico por meio de endoscopia em cavalos de pólo a fim de avaliar a ocorrência de HPIE nestes equinos. Para tal, foram utilizados 44 equinos, regularmente utilizados em competições de pólo no Rio de Janeiro. A endoscopia foi realizada entre 30 e 90 minutos após a competição, sendo aplicado o escore para quantificação do sangramento. A ocorrência da HPIE foi de 34,09\% (15/44) sendo que 73,33\% (11/15) apresentaram grau 1 de sangramento, $6,67 \%(01 / 15)$ apresentaram grau 2 de sangramento seguido de $20 \%(3 / 15)$ dos equinos com grau 3. No presente trabalho não foi observada relação estatisticamente significativa entre a presença de HPIE e o sexo, assim como não houve relação estatisticamente significativa entre animais com secreção traqueal e animais positivos para a HPIE. Conclui-se que existe a ocorrência do sangramento e a sua intensidade é menor quando comparamos animais que praticam polo frente aos que são destinados às corridas.
\end{abstract}

Palavras-chave: cavalo de pólo, endoscopia, HPIE

\section{OCCURRENCE OF PULMONARY HEMORRHAGE INDUCED BY EXERCISE IN POLO HORSES IN THE CITY OF RIO DE JANEIRO / RJ}

\begin{abstract}
Polo is one of the most ancient horse sports and was introduced in Brazil in the twenties. Respiratory diseases are considered the second main cause of poor performance, leading to significant economic losses in race horses. Exercise induced pulmonary hemorrhage (EIPH) is one of these diseases and affects 40 to $90 \%$ of race horses, however there are few researches directed to polo horses. The matter is of great concern to the equine industry due to the financial implications that result from poor performance, loss of training days, the need of medication before the races and even withdrawal of the animals from competitions. The endoscopic examination using a score is the diagnostic method most frequently used to quantify hemorrhage intensity. The main objective of this project was to do a clinic-epidemiological study using respiratory endoscopy in polo horses to evaluate the occurrence of EIPH. Respiratory endoscopy was performed between 30 to 90 minutes after the competition. This research studied 44 polo horses from Itanhangá Golf Club, located at Barra da Tijuca, Rio de Janeiro and from Regimento de Cavalaria de Guardas Andrade Neves (RCGd/EB), located in Deodoro, Rio de Janeiro, were used. From the 44 polo horses studied, $34.09 \%$ (15 animals) showed some degree of EIPH at endoscopic examination. There was no statistically significant relation between the occurrence of EIPH and gender. There was also no statistically significant relation between tracheal secretion and EIPH. Therefore, it is concluded that EIPH occurs in polo horses and EIPH's intensity is lower compared to thoroughbred horses.
\end{abstract}

Key Words: polo horses, endoscopy and EIPH 


\section{INTRODUÇÃO}

O aparelho respiratório é fundamental para a saúde e bom desempenho atlético dos equinos, sendo os processos mórbidos neste sistema responsáveis por prejuízos orgânicos e econômicos consideráveis nesta espécie.

A HPIE é definida como sendo a presença de sangue livre, de origem pulmonar, na árvore traqueobrônquica ou sinais de sangue, após exercício intenso. (Costa et al., 2004; Newton et al., 2005). Para o diagnóstico com maior possibilidade de observação, o exame endoscópico é o melhor procedimento e deve ser realizado entre 30 e 90 minutos após o exercício (Sweeney \& Reilly, 2001). De acordo com Derksen e Robinson (2002), esta é uma das enfermidades de maior importância do trato respiratório equino, juntamente com a Obstrução Recorrente das Vias Aéreas (ORVA) e a Doença Inflamatória das Vias Aéreas (DIVA).

$\mathrm{Na}$ avaliação por endoscopia para diagnóstico dessa enfermidade, observou-se que 40 a $90 \%$ dos cavalos de corrida apresentam a HPIE (Pascoe et al., 1981; Raphael \& Soma, 1982).

Biava et al. (2007) descreveram a enfermidade em quartos de milha sendo que $40 \%$ dos cavalos participantes de provas de seis balizas e $75 \%$ nos participantes de prova de três tambores apresentaram o problema.

Sweeney (1991) encontrou cerca de $11 \%$ em cavalos de pólo com algum grau de HPIE. De acordo com Moran et al. (2003), em um estudo sobre a ocorrência de HPIE em cavalos de pólo no Chile, $46 \%$ dos animais estudados eram positivos para HPIE.

Apesar do crescimento do número de animais utilizados para a atividade de pólo e a alta frequência já relatada das enfermidades respiratórias em equinos (Sweeney, 1991; Chapman et al. 2000; Lessa et al. 2005), os animais utilizados para a prática do pólo ainda são pouco estudados e pouco explorados no que se refere a estas doenças.

No Brasil, existem poucos estudos a cerca da ocorrência da HPIE em equinos (Costa et al., 2004) e não foram observados até 0 momento estudos de ocorrência desta enfermidade por diagnóstico endoscópico em animais de pólo.

Desta forma, o presente trabalho teve como objetivo verificar a ocorrência e intensidade da HPIE em cavalos de pólo no Rio de Janeiro, avaliar a influência do sexo na ocorrência da enfermidade, assim como, se a presença de HPIE favorece a presença de secreção traqueal.

\section{MATERIAL E MÉTODOS}

Foram utilizados 44 equinos adultos, 19 machos e 25 fêmeas, mestiços da raça crioulo com purosangue inglês, de idade entre três e 21 anos, regularmente utilizados em jogos de pólo na cidade do Rio de Janeiro. Os animais foram alimentados com ração comercial, aveia e volumoso (feno de alfafa) e suplementados com sal mineral. Todos os animais foram mantidos em cocheiras e periodicamente vermifugados e vacinados contra tétano, influenza, encefalomielite leste, oeste e raiva. Todos os equinos estudados jogaram apenas um tempo da partida de pólo com duração de sete minutos.

Cada animal foi submetido a uma única avaliação endoscópica do trato respiratório, que ocorreu em tempo que variou entre 30 e 90 minutos após o término do tempo no qual jogou, segundo a metodologia preconizada por Pascoe et al. (1981).

O exame endoscópico foi realizado utilizando-se um Colonofibroscópio Olympus modelo CF 10L, acoplado a uma Microcâmera Karl 
Storz modelo Endovision XL 20280130 NTSC e uma Filmadora Digital Sony modelo DCR HC-65. Todas as endoscopias foram digitalizadas, armazenadas e analisadas posteriormente sempre pelo mesmo examinador.

Foram realizadas avaliações semi-quantitativas para a presença de sangue, segundo Costa et al. (2004) e para a presença de muco, segundo Gerber et al. (2004).

As variáveis não-paramétricas (sexo, escore de secreção traqueal e a HPIE) foram submetidas ao teste de Kruskal-Wallis com nível de significância $5 \% \quad(p<0,05)$, utilizando-se o programa The SAS System ${ }^{\circledR}$.

\section{RESULTADOS E DISCUSSÃO}

Todos os animais apresentaram os parâmetros do exame físico dentro da normalidade de acordo com McGorum et al. (2007).

Dos 44 equinos de pólo estudados, $\quad 34,09 \% \quad(15 / 44)$ apresentaram algum grau de HPIE durante o exame endoscópico, dos quais $73,33 \% \quad(11 / 15)$ grau 1 de sangramento, 6,67\% (01/15) grau 2 de sangramento seguido de $20 \%$ (3/15) dos equinos sangradores diagnosticados com grau 3 de HPIE. Não foram observados graus 4 e 5 de HPIE durante o estudo.

Em um estudo sobre a ocorrência de HPIE em cavalos de pólo no Chile, Moran et al. (2003) observaram que $46 \%$ dos animais estudados eram positivos para HPIE. Já Lessa et al. (2005) citam que $11 \%$ dos equinos de pólo apresentam algum grau de HPIE. Não existem muitos estudos sobre a ocorrência de HPIE em cavalos de pólo, desta forma, os poucos trabalhos existentes apresentam valores distintos como os citados anteriormente.
O valor encontrado para a ocorrência da HPIE no presente trabalho está próximo à porcentagem encontrada por Moran et al. (2003), porém, ainda, inferior. Acredita-se que isso se ocorra em função do momento em que a endoscopia foi realizada. No presente trabalho, o exame endoscópico foi realizado entre 30 e 90 minutos após o exercício, ao passo que no trabalho chileno, a endoscopia ocorreu duas a quatro horas após o exercício. Tanto o nosso trabalho quanto o trabalho de Moran et al. (2003) não apresentaram HPIE com graduação superior ao grau 3.

O pólo é uma atividade desportiva de menor intensidade aeróbia, uma vez que o animal não é forçado ao seu limite por um trajeto tão longo quanto o de uma corrida de cavalos. E, por isso, quando comparado ao turfe, requer menos da capacidade cardio-respiratória do animal, exigindose menos deste equino durante a atividade física e consequentemente, menor a hipertensão pulmonar gerada e menor a HPIE.

A análise das variáveis: sexo, presença de secreção traqueal e de HPIE pelo método de Kruskal-Wallis revelou não existir relação estatisticamente significativa entre elas.

Embora os dados avaliados neste estudo não permitam afirmar que haja predisposição à HPIE em função do sexo, $60 \%$ dos animais positivos para HPIE foram machos castrados e, portanto, $40 \%$ restantes fêmeas. Estes dados corroboram com Moran et al. (2003), que observaram em seu estudo com cavalos de pólo que $63,6 \%$ dos animais positivos para HPIE eram machos e $36,4 \%$ eram, consequentemente fêmeas, e ainda, estão de acordo com o observado por Pascoe (1991).

Observa-se uma incidência mais alta em machos castrados do que em 
éguas e em machos inteiros e este evento pode se relacionar com a diminuição da eritropoiese e síntese de hemoglobina e por uma concentração menor de testosterona (Robertson e Rooney, 1997; Pascoe,2000). As éguas (73\%) e os machos castrados (74\%) são mais acometidos quando comparados a garanhões (49\%) (Hillidge e Whitlock, 1986), embora pareça não haver relação entre HPIE e o sexo dos animais (Rains et al., 2003; Hinchcliff et al., 2004; Baccarin, 2005).

A relação entre a HPIE e a inflamação já foi demonstrada anteriormente (McKane et al., 1993) e foi considerada significativa no estudo epidemiológico de Newton e Wood (2002), apesar dos mecanismos pelos quais esta ocorre ainda serem apresentados de forma hipotética, como no recente estudo de Derksen et al. (2009).

Porém, no presente trabalho não houve relação estatisticamente significativa entre animais com algum grau de secreção traqueal e animais positivos para a HPIE. De acordo com Michelotto (2010), observou-se um percentual de HPIE (determinado pela presença de hemossiderófagos no LBA) maior que o de inflamação pulmonar ( $>5 \%$ de neutrófilos e/ou $>1 \%$ de eosinófilos na avaliação citológica diferencial do lavado broncoalveolar). Portanto a HPIE pode preceder a inflamação no pulmão dos cavalos de corrida, tal como foi sugerido por Derksen et al. (2009), mas que requer outras investigações para excluir que o contrário estivesse acontecendo, isto é, um processo inflamatório em período anterior do treinamento que pudesse ter ocasionado alguma fragilidade pulmonar.

As relações entre hemorragia e secreção traqueal estão apresentadas nas tabelas 1 e 2 .
Tabela 1 - Relação entre os escores endoscópicos de secreção traqueal e HPIE dos 44 equinos examinados. Rio de Janeiro - RJ, 2010.

\begin{tabular}{|l|l|l|l|l|l|}
\hline & \multicolumn{3}{|l|}{ G raduação da HPIE } & \\
\hline $\begin{array}{l}\text { Secreção } \\
\text { Traqueal }\end{array}$ & 0 & 1 & 2 & 3 & $n / \%$ \\
\hline 0 & 11 & 03 & Zero & 03 & $17 / 38,64$ \\
\hline 1 & 13 & 04 & Zero & Zero & $17 / 38,64$ \\
\hline 2 & 05 & 04 & 01 & Zero & $10 / 22,73$ \\
\hline $\mathbf{n} / \%$ & $29 / 65,91$ & $11 / 25,00$ & $01 / 2,27$ & $03 / 6,82$ & $44 / 100$ \\
\hline
\end{tabular}

Tabela 2 - Animais examinados por endoscopia do trato respiratório após o secreção traqueal e HPIE dos 44 equinos examinados. Rio de Janeiro-RJ, 2010.

\begin{tabular}{|l|l|l|l|}
\hline & HPIE + & HPIE - & Total \\
\hline Secreção + & 09 & 18 & 27 \\
\hline Secreção - & 06 & 11 & 17 \\
\hline Total & 15 & 29 & 44 \\
\hline
\end{tabular}

\section{CONCLUSÃO}

A ocorrência da HPIE em cavalos de pólo na cidade do Rio de Janeiro é de 34,09\%. O exame endoscópico torna-se, desta forma, uma ferramenta útil ao médico veterinário na investigação clínica sobre enfermidades respiratórias e à diminuição do desempenho atlético, assim como auxilia a definir prognósticos. O sexo não influenciou na presença e na intensidade de HPIE. Machos e fêmeas são igualmente afetados sob o ponto de vista estatístico.

Quanto à relação entre a HPIE e o processo inflamatório, este estudo não pôde comprovar se animais inflamados estão mais susceptíveis ao sangramento.

\section{NOTAS INFORMATIVAS}

Este trabalho de pesquisa foi realizado em acordo com os Princípios Éticos na Experimentação Animal do Colégio Brasileiro de Experimentação Animal (COBEA), sendo aprovado pelo Comitê de Ética de Pesquisa com Animais (CEPA) do Núcleo de Animais de Laboratório (NAL) da UFF sob 0 número de registro 0068-08.

\section{REFERÊNCIAS}


BACCARIN, R. Y.A. Diagnóstico e tratamento das pneumopatias de esforço. II Simpósio Internacional do Cavalo Atleta e IV Semana do Cavalo - SIMCAV. Anais... Belo Horizonte, Minas Gerais, 2005, p.12-28.

BIAVA, J.S.; GONÇALVES, R.C.; DORNBUSCH, P.T. et al. Avaliação clínica e citológica do trato respiratório de cavalos da raça quarto de milha, após o exercício.

Archives of veterinary science, v.11, n.1, p.6065, 2007.

BIRKS, E.K.; MATHIEU-COSTELLO, O.; FU, Z. et al. Very high pressures are required to cause stress failure of pulmonary capillaries in Thoroughbred racehorses. Journal of Applied Physiology, v.82, n.5, p.1584-1592, 1997.

COSTA,M.F.M.;THOMASSIAN,A.;GOMES,T.S. et al. Estudo da Hemorragia Pulmonar induzida por esforço (HPIE) em cavalos de corrida PSI através da análise de 1889 endoscopias respiratórias após corrida. Revista Brasileira de Ciência Veterinária, v.11, n.3, p.8991,2004.

CHAPMAN, P.S.; GREEN,C.; MAIN,J.P.M. et al. Retrospective study of the relationships between age, inflammation and isolation of bacteria from the lower respiratory tract of thoroughbred horses. The Veterinary Record, v.146, n.4, p.91-91,2000.

DERKSEN, F.J.; ROBINSON, N.E. Overview of the equine respiratory system. In: LEKEUX, P. Equine Respiratory Disease. Ithaca: International Veterinary Information Service, 2002. Disponível em:

<http://www.ivis.org/special_books/Lekeux/>. Acesso em: abril 2009

GERBER, V.; STARUB, R.; MARTI, E. et al. Endoscopic scoring of mucus quantity and quality: observer and horse variance and relationship top inflammation, mucus viscoelasticity and volume. Equine Veterinary Journal, v.36, n.7, p.576-582, 2004.

HILLIDGE, C.J.; WHITLOCK, T.W. Sex variation in the prevalence of exerciseinduced pulmonary haemorrhage in racing quarter horse. Research in Veterinary Science, v.40, p.406-407, 1986.

HINCHCLIFF, K.W.; KANEPS, A.J.; GEOR, R.J. Equine sports medicine and surgery. Philadelphia: W. B. Saunders Company, 2004. $705 \mathrm{p}$.
LESSA, D.A.B.; MACHADO, C.H.; DUARTE, C. $S$. et al. Enfermidades do Trato Respiratório Posterior em Equinos de Equitação no Rio de Janeiro: Prevalência e Aspectos ClínicoLaboratoriais. Revista Brasileira de Ciência Veterinária, v.12, n.1/3, p.77-83, 2005.

McGORUM, B.C.; DIXON, P.M. Clinical examination of the respiratory tract. In: McGORUM, B.C. et al. J. Equine Respiratory Medicine and Surgery. Philadelphia: Elsevier, 2007, p.103-117.

McKANE, S.A.; CANFIELD, P.J.; ROSE, R.J. Equine bronchoalveolar lavage cytology: survey of thoroughbred racehorses in training. Australian Veterinary Journal, v.70, n.11, p.401-404, 1993.

MORAN, G.; CARRILLO,R.; CAMPOS, B. et al. Evaluacion endoscopica de hemorragia pulmonar inducida por el ejercicio en equinos de polo. Archivos-de-Medicina-Veterinaria, v.35, n.1, p.109-113, 2003.

NEWTON, J.R.; WOOD, J.L. Evidence of an association between inflammatory airway disease and EIPH in young Thoroughbreds during training. Equine Veterinary Journal, v.34, supl., p.417-424, 2002.

NEWTON, J.R.; ROGERS, K.; MARLIN, D.J. et al. Risk factors for epistaxis on British racecourses: evidence for locomotory impactinduced trauma contributing to the aetiology of exercise-induced pulmonary haemorrhage. Equine Veterinary Journal, v.37, p.402-411, 2005.

PASCOE, J.R.; FERRARO, G.L.; CANNON, J.H. et al. Exercise-Induced Hemorrhage in racing Thoroughbreds:A preliminary study. Am Journal of Veterinary Research, v.42, p.703-707, 1981.

PASCOE, J. R. Exercise induced pulmonary hemorrhage, In: Beech, J. Equine Resp. Disorder. Ed. Lea and Febiger, Pennsylvania, USA. 1991.

PASCOE, J. 2000. Text reprinted with permission from proceeding of the American Association of Equine Practitioners. XI Congreso Nacional de Medicina Veterinaria. Anais... Santiago de Chile.

RAINS, J.R.; COLLIER, M.A.; MACALLISTER, C.G. Epistaxis/exercised induced pulmonary 
hemorrhage in horses. Disponível em: <www. mrhorse.com>. Acesso em: 2-fev-2003.

RAPHAEL, C.F.; SOMA, L.R. Exercise-induced pulmonary hemorrhage in Thoroughbreds after racing and breezing. American Journal of Veterinary Research, v.43, n.7., p.1123-1125, 1982.

ROBERTSON J. L.; J. R, ROONEY. The pathology of the equine respiratory system. Dubai international equine symposium.

Anais...Dubai, Neyenesch Printers Inc. p.p. 189-239, 1997.

SWEENEY, C.R. Exercise-Induced Pulmonary Hemorrhage. Veterinary clinics of North America Equine practice, v.7, n.1, 1991.

SWEENEY, C. R. \& REILLY, L. K. Sistema Respiratório. In_ : Segredos em Medicina de Equinos. Porto Alegre: Artmed, 2001, p.136151. 